\title{
Integrated assessment of the direct and indirect effects of resource gradients on tree species recruitment
}

\author{
INÉS IBÁÑEZ ${ }^{1,3}$ AND SARAH MCCARTHY-NEUMANN ${ }^{1,2}$ \\ ${ }^{1}$ School of Natural Resources and the Environment, University of Michigan, Ann Arbor, Michigan 48109 USA \\ ${ }^{2}$ Department of Forestry, Michigan State University, East Lansing, Michigan 48824 USA
}

\begin{abstract}
Understanding the dynamics of tree establishment is critical to assess forests' composition, management practices, and current responses to global change. We carried out a field seedling transplant experiment to assess not only the direct effects of resources influencing recruitment of four tree species, but also their indirect and combined effects. Our analysis integrated first growing season demographic data together with estimates of mycorrhizal fungal colonization and resource availability (light, soil moisture, and soil nitrogen). Only by considering both the direct and indirect effects of resources we were able to account for most of the variability observed during seedling recruitment. Contrary to expectations, increasing light levels were not always beneficial for recruitment even in low light habitats, and soil moisture availability benefited seedling growth but not survival. In addition, mycorrhizal fungal colonization was not always favored by high light levels or by increasing soil moisture. Seedling survival for all species was lower in plots with higher arbuscular mycorrhizal fungi, while the association with ectomycorrhizal fungi varied from beneficial to detrimental. When integrating the direct, indirect, and interactive effects of resource availability and mycorrhizal fungal colonization on tree recruitment dynamics we found that species responded in a nonlinear fashion to increasing resource levels, and we also identified thresholds, i.e., shifts in the direction of the response, along the resource gradient. Our integrated assessment considerably outperformed a null model where only direct effects of resources were accounted for. These results illustrate how the combination of direct, indirect, and combined effects of driving variables better represents the complexity of the processes determining tree species recruitment than simple resource availability mechanisms.
\end{abstract}

Key words: Acer saccharum; Carya glabra; hierarchical Bayesian analysis; irradiance; nitrogen; Nyssa sylvatica; Quercus rubra; recruitment; temperate forests.

\section{INTRODUCTION}

In forests, recruitment of tree species (their establishment and survival during the first few years) is one of the most critical processes determining the structure of the entire ecosystem (Grubb 1977). Lack of sufficient recruitment could affect a species likelihood of persisting, and ultimately impact community composition and ecosystem function (e.g., Gurevitch et al. 2006, Suarez and Kitzberger 2008). Despite the numerous variables, interactions, and feedbacks influencing the recruitment of new individuals, methodological constraints allow us to only consider a small fraction of these factors at a time, precluding our understanding of the complex dynamics behind the recruitment process. Still, we can heighten our insight into the dynamics underlying recruitment by integrating the available data sets into a comprehensive analysis. An integrated approach will take into account not only the direct effects, but also the feedbacks, indirect effects, and interactions that drive recruitment dynamics.

Manuscript received 11 April 2013; revised 17 July 2013; accepted 17 July 2013. Corresponding Editor: A. W. D'Amato.

${ }^{3}$ E-mail: iibanez@umich.edu
Most studies of tree species recruitment focus on resource availability (e.g., Beckage et al. 2000, Comita et al. 2009), and, when only one or a few variables are being considered (e.g., light, water), assumptions of linear responses and simple relationships between variables are the norm (e.g., Kaelke et al. 2001, Ibáñez et al. 2007, 2008). However, the complexity of the interactions among variables and their combined effects likely modify species responses in such a way that thresholds and nonlinear trends could prevail (e.g., Ackerly and Bazzaz 1995, Bereau et al. 2005, Uriarte et al. 2012). Even if considered independently, all these drivers act simultaneously; the recruitment niche defined with respect to one variable is going to be highly dependent on the environmental context determined by many other variables. For example, in the case of tree seedlings, increasing light levels will only be beneficial if soil water content is sufficient and if competition from the ground vegetation is relatively low. This is because habitats with high light are also associated with higher evaporation rates and higher competition from forbs and grasses (e.g., Caldwell et al. 1995, Löf 2000). Thus, for some tree species, an increasing light environment may switch from being 
beneficial to detrimental at a certain threshold. Also, the nature of mycorrhizal fungal symbioses on tree seedlings, positive or negative, or the effect of pathogens, lethal or not, could strongly depend on the light environment as light levels determine carbon assimilation rates and thus the ability of seedlings to sustain positive or neutral interactions (Johnson et al. 1997).

If we aim to understand and predict tree recruitment in forest ecosystems, we will need to consider the multiple processes underlying recruitment patterns and their catalysts, i.e., the driving variables and their interacting effects. The combination of key demographic elements can help us accomplish this goal, since demographic dynamics are the product of all the processes taking place during recruitment, and integrate all direct and indirect effects of the biotic and abiotic environment on performance of individuals (O'Connor et al. 2012).

In this study, we used extensive demographic data on seedling survival, growth and mycorrhizal colonization of the roots together with information on the abiotic environment (light levels, soil water availability, and soil nutrients) to assess recruitment dynamics of four temperate tree species. We used a hierarchical Bayesian framework to develop an integrated analysis identifying the direct and indirect pathways by which resources affect recruitment dynamics. To accomplish this, we did the following: (1) Analyzed seedling survival as a function of extrinsic factors, i.e., light and soil moisture, resources well known to affect recruitment patterns (e.g., Ibáñez et al. 2007); and of the intrinsic variables, i.e., mycorrhizal colonization and growth rates, as they determine the health status of the seedlings and consequently affect survival (Myers and Kitajima 2007, Piper et al. 2009). (2) Evaluated seedling growth rates as a function of mycorrhizal fungal colonization, as this may have facilitated growth by providing additional resources (Bereau et al. 2005, Teste et al. 2009), and as a function of light levels, soil moisture, soil nitrogen content, and initial plant height. (3) Estimated colonization probability and abundance of arbuscular mycorrhizal (AMF) and ectomycorrhizal (EMF) fungi on seedling roots as a function of the abiotic environment and of the initial size of the seedling (a proxy for seed reserves and initial available carbon [Zangaro et al. 2005, Jin et al. 2009]).

We then used the outcomes from the integrated model to quantify seedling survival along environmental gradients when not only the direct effects, but also the indirect effects (through the effects of growth and mycorrhizal fungi on survival) of the resources were also considered.

\section{Methods}

We conducted a field transplant experiment to study the recruitment of four common tree species in eastern North American temperate forests. We established experimental plots and carried out work in the summer of 2009 and then replicated the experiment in 2010.

Study sites, experimental setup, and environmental sampling.-The experiment was carried out in the Great Lakes region, north-central United States, at two latitudes (north and south) that differed mainly in the length of the growing season and major forest types (Table 1). At each location, we worked in representative forest types and, within each forest type, we set up experimental plots $(5 \times 5 \mathrm{~m})$ in two habitats (i.e., under the forest canopy [canopy] and in canopy gaps [gap]). We had a total of 28 plots ( 2 latitudes $\times 3$ forest types $\times 2$ habitats $\times$ 2-4 replicates) representing a variety of environmental conditions and biotic environments (see Appendix A for thorough description of the sites' conditions).

At each plot, we collected five soil samples $(5 \mathrm{~cm}$ diameter $\times 10 \mathrm{~cm}$ depth) that were aggregated for an analysis of soil characteristics $(\mathrm{pH}$, inorganic nitrogen [nitrate and ammonium], total nitrogen, phosphate, potassium, calcium, and magnesium [McCarthy-Neumann and Ibáñez 2012]). Each summer, soil moisture was measured with a portable soil moisture meter $(\mathrm{HH} 2$; Delta-T, Cambridge, UK) at five points (10 cm in depth) in each plot every two weeks throughout the duration of the experiment $(\sim 12$ weeks). We used each summer's average of those measurements to characterize the soil's water availability associated with each plot and year. Light levels were estimated using canopy photos (Rich et al. 1993). After the canopy had fully developed (mid July), we took photos at $0.5 \mathrm{~m}$ above the ground at the center and four corners of each plot using an 8-mm fisheye lens, once a year. From these photos, the percentage of full sun reaching the forest floor, as an average of the five points, was calculated using Hemiview software (Delta-T, Cambridge, UK).

Seedling transplants.-We worked with four tree species that vary in their ecological traits, their distributional range, and in their phylogenetic origins (Table 2). We used local wild sources of seeds from locations around our two study regions. Each Spring we germinated the seeds in containers filled with potting soil (Metro-Mix 380; SunGro Horticulture, Agawam, Massachusetts, USA) at the University of Michigan greenhouses. Approximately 3-4-week-old seedlings were transplanted into each field plot (20 seedlings/species) early in the growing season, May-June. Seedlings were planted at $25-\mathrm{cm}$ interval rows imbedded with the natural ground vegetation. Individual seedling height and diameter were measured before planting to account for potential size effects.

We recorded survival two weeks after planting to exclude mortality due to transplant shock from the analysis, and again at the end of the summer to estimate plot-level summer survival. Among those seedlings that survived, we harvested a maximum of three (per plot and species). Prior to drying the seedlings, root fractions, 5-10 $1-\mathrm{cm}$ sections of wet root, were retained, weighed, and stained with $5 \%$ 
TABLE 1. Locations in the Great Lakes region and habitat types (dominant tree species and their primary mycorrhizal fungi associations as adults [AMF, arbuscular mycorrhizal fungi; EMF, ectomycorrhizal fungi]) for the transplant experiment.

\begin{tabular}{|c|c|c|}
\hline Site features & North $\left(45^{\circ} 36^{\prime} \mathrm{N}, 84^{\circ} 41^{\prime} \mathrm{W}\right)$ & South $\left(42^{\circ} 28^{\prime} \mathrm{N}, 84^{\circ} 00^{\prime} \mathrm{W}\right)$ \\
\hline $\begin{array}{l}\text { Temperature ranges } \\
\text { Soil textures }\end{array}$ & $\begin{array}{l}-14^{\circ} \mathrm{C} \text { and } 26^{\circ} \mathrm{C} \\
\text { gravelly sandy, loamy }\end{array}$ & $\begin{array}{l}-8.8^{\circ} \mathrm{C} \text { and } 28.8^{\circ} \mathrm{C} \\
\text { sandy loams }\end{array}$ \\
\hline \multicolumn{3}{|l|}{ Forest types } \\
\hline $\begin{array}{l}\text { Stand } 1 \text { (north, } 4 \text { plots); } \\
\text { stand } 4 \text { (south, } 4 \text { plots) }\end{array}$ & $\begin{array}{l}\text { northern hardwoods } \\
\text { (Fagus grandifolia } \text { EMF, } \\
\text { Quercus rubra } \text { EMF, Acer saccharum AMF) }\end{array}$ & $\begin{array}{l}\text { white and red oak } \\
\text { (Q. alba EMF, Q. rubra, A. saccharum) }\end{array}$ \\
\hline $\begin{array}{l}\text { Stand } 2 \text { (north, } 4 \text { plots); } \\
\text { stand } 5 \text { (south, } 6 \text { plots) }\end{array}$ & $\begin{array}{l}\text { pine-aspen } \\
\text { (Pinus strobus EMF, } \\
\text { Populus grandidentata EMF and AMF, Q. rubra) }\end{array}$ & $\begin{array}{l}\text { red oak-maple } \\
\quad(Q . \text { rubra, A. saccharum })\end{array}$ \\
\hline $\begin{array}{l}\text { Stand } 3 \text { (north, } 4 \text { plots); } \\
\text { stand } 6 \text { (south, } 6 \text { plots) }\end{array}$ & $\begin{array}{l}\text { aspen } \\
\quad(P . \text { grandidentata }, Q . \text { rubra }, F . \text { grandifolia })\end{array}$ & $\begin{array}{l}\text { black oak-hickory } \\
\text { (Q. velutina } \mathrm{EMF}, \text { Carya glabra } \mathrm{EMF})\end{array}$ \\
\hline
\end{tabular}

Notes: Temperature ranges indicate average minimum (January) and maximum (July) records. Elevation along the sites ranges from $180 \mathrm{~m}$ to $525 \mathrm{~m}$. Mycorrhizal fungi associations are only reported the first time a species appears.

Schaeffer black ink in vinegar solution (Vierheilig et al. 1998) to assess mycorrhizal colonization. The percentage of root colonized by arbuscular mycorrhizal fungi (AMF) was quantified by inspecting 100 intersections between the microscope eyepiece cross hair for AMF structures (i.e., vesicles, arbuscules, coils, and hyphae that were near but not at vesicles or arbuscules) every $1 \mathrm{~mm}$ at $200 \times$ magnification (McGonigle and Fitter 1990). The percentage of root colonized by ectomycorrhizal fungi (EMF) was quantified by counting the number of intact root tips with and without Hartig nets at $100 \times$ magnification every $2 \mathrm{~mm}$ along the root until 100 root tips had been scored. Remaining root, stem, and leaf fractions were dried in a forced-air oven at $70^{\circ} \mathrm{C}$ for biomass measurements. Total dry root biomass was derived using the mass of the total wet root material and the wet: dry mass ratios determined from root samples. To standardize our measurements for comparisons, each individual's average daily growth rate was estimated from the total dry biomass divided by the number of days growing in the field $(\mathrm{mg} / \mathrm{d})$.

Analysis.-Prior to the analyses, we performed extensive exploration of the data to identify the strongest relationships between our variables (see Appendix B). We developed a series of models that reflected our knowledge of the system (e.g., saturating response of growth rates to light) and the results from the exploratory analysis, e.g., different response to light between the two habitats, or nitrogen (total) being the soil nutrient with highest correlations with our demographic data. We could have also tried interaction effects between variables, for example, between light and soil moisture, but we did not because the data did not cover all possible combinations of the different variables included, e.g., there were not plots at high light and high moisture. We report on the final submodels; these are the models that best fit the data (based on posterior predicted loss [Gelfand and Ghosh 1998]). Prior to the analyses initial seedling height for each species and soil total nitrogen for each plot were standardized ([value - mean]/SD).

Mycorrhizal fungal colonization.-AMF or EMF root colonization data were analyzed independently using a zero-inflated Poisson model to account for the large proportion of non-colonized seedlings. The probability of not detecting colonization, $\varphi$, was integrated into a count process by differentiating zero counts from the rest (Zuur et al. 2009). The percentage of AMF or EMF, $M_{i}$, in seedling $i$, $\operatorname{plot}(i)$, year $(i)$, and habitat $(i)$ was estimated from a Poisson likelihood with mean $\lambda_{i}$ :

$$
P(M=m)=\left\{\begin{array}{cc}
\varphi+(1-\varphi) e^{-\lambda} & \text { for } m=0 \\
(1-\varphi) \frac{1}{m !} \lambda^{m} e^{-\lambda} & \text { for } m>0
\end{array}\right.
$$

and process models,

TABLE 2. List of studied species, with family and number of seedlings examined for mycorrhizal colonization and seedling biomass in parentheses, average seed size (mg), their shade tolerance status as seedlings (Barnes and Wagner 2007), growth status (Barnes and Wagner 2007), symbiotic associations with seedlings, and locations where they are native in our study sites.

\begin{tabular}{lcclcc}
\hline \hline \multicolumn{1}{c}{ Species } & Seed size $(\mathrm{mg})$ & Shade tolerance & Growth & Mycorrhizae & Native \\
\hline Acer saccharum $\dagger$ (Sapindaceae, 134) & 64.9 & very tolerant & very slow & AMF & north and south \\
Nyssa sylvatica (Cornaceae, 88) & 153.8 & tolerant & slow & AMF & south \\
Carya glabra (Juglandaceae, 108) & 2272 & intermediate & slow & AMF and EMF & south \\
Quercus rubra (Fagaceae, 199) & 4127 & intermediate & moderate fast & AMF and EMF & north and south \\
\hline
\end{tabular}

$\dagger$ A. saccharum seeds for 2010 were collected from wild populations in Pennsylvania due to lack of local seeds that year (purchased from Sheffield's Seed Company, Locke, New York, USA). 
$\operatorname{logit}(\varphi) \sim \operatorname{Normal}\left(\omega 1_{\text {plot }(i), \operatorname{year}(i)}+\omega 2\right.$ plant height $\left._{i}, \sigma_{\varphi}^{2}\right)$

$$
\begin{aligned}
\log \left(\lambda_{i}\right) \sim & \operatorname{Normal}\left(\alpha 1_{\text {plot }(i), \operatorname{year}(i)}+\alpha 2_{\text {habitat }(i)} \operatorname{light} \operatorname{plot}(i), \text { year }(i)\right. \\
& \left.+\alpha 3 \text { soil moisture } \operatorname{plot}(i), \operatorname{year}(i), \sigma_{\lambda}^{2}\right)
\end{aligned}
$$

We included individual random effects, with variances $\sigma_{\varphi}^{2}$ and $\sigma_{\lambda}^{2}$, and a random effect for each plot and year, $\omega 1_{\text {plot,year }}$ and $\alpha 1_{\text {plot,year, with means and vari- }}$ ances $\omega 1_{\mathrm{m}}, \alpha 1_{\mathrm{m}}, \sigma_{\omega}^{2}$ and $\sigma_{\alpha}^{2}$, reflecting the particular mycorrhizal fungal community associated with that plot and year (for which we did not have any specific information).

Growth rates.-Daily growth rate, Growth, was analyzed as a saturating function of light (a major limiting resource in these forests [Canham et al. 1999]) and a linear function of soil moisture (Eq. 3). This is a well-established approach for this type of data (e.g., Clark et al. 2003, Ibáñez et al. 2009) with likelihood

$$
\operatorname{Growth}_{i} \sim \operatorname{Normal}\left(G_{i}, \sigma_{\mathrm{G}}^{2}\right)
$$

and process model

$$
\begin{aligned}
G_{i}= & \operatorname{gmax}_{i} \frac{\operatorname{light}_{\text {plot }(i), \text { year }(i)}-\mathrm{lo}_{i}}{\operatorname{light}_{i \operatorname{plot}(i), \text { year }(i)}+\theta_{i}} \\
& +\delta \text { soil moisture }_{\text {plot }(i), \text { year }(i)} .
\end{aligned}
$$

We then used the results for the exploratory data analysis (see Appendix B for a thorough description) to estimate the parameters of the curve, gmax, lo, and $\theta$, as a function of additional information collected on the seedlings and plots:

$$
\begin{aligned}
\operatorname{gmax}_{i} & \sim \operatorname{Normal}\left(\gamma 1+\gamma 2 \text { plant height }_{i}, \sigma_{\text {gmax }}^{2}\right) \\
\operatorname{lo}_{i} & \sim \operatorname{Normal}\left(\kappa 1+\kappa 2 \operatorname{Nitrogen}_{\operatorname{plot}(i)}, \sigma_{\mathrm{lo}}^{2}\right) \\
\theta_{i} & \sim \operatorname{Normal}\left(\mu 1+\mu 2 M_{\mathrm{AMF} i}+\mu 3 M_{\mathrm{EMF} i}, \sigma_{\theta}^{2}\right)
\end{aligned}
$$

where $\operatorname{gmax}_{i}$ (Eq. 4) represents the maximum growth rate for an individual, modeled as a function of a species-level constant $(\gamma 1)$, the individual's initial plant height (this reflects the effect of seed reserves, which might influence growth rates), and of individual random effects (with variance $\sigma_{\text {gmax }}^{2}$ ). The compensation point (Eq. 5), minimum amount of light necessary to start growth (lo), was estimated as a function of a specieslevel constant $(\kappa 1)$, plot's total nitrogen, and individual random effects (with variance $\sigma_{\mathrm{lo}}^{2}$ ). The half-saturation parameter (Eq. 6), amount of light necessary to reach of the maximum growth rate $(\theta)$, was calculated as the combination of a species-level parameter $(\mu 1)$, the effects of percentage of mycorrhizal fungal colonization (as the mycorrhizal symbiosis could have affected the level of light at which the seedlings reach their maximum photosynthetic potential), and of individual random effects (with variance $\sigma_{\theta}^{2}$ ).
Using parameter values from the mycorrhizae and growth rate sub-models, we estimated plot-level predicted percentage of mycorrhizal fungal colonization $\left(M_{\mathrm{P}-\mathrm{AMF}}\right.$ and $\left.M_{\mathrm{P} \text {-EMF }}\right)$ and growth rates $\left(G_{\mathrm{P}}\right)$ for each of our experimental plots and years.

Survival.-Survival data from each summer were analyzed using the early-summer counts of seedlings,

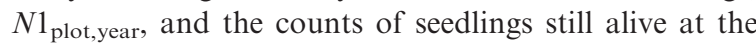
end of the summer, $N 2_{\text {plot,year. }}$ We used a binomial likelihood with probability of survival $p_{\text {plot,year: }}$ :

$$
N 2_{\text {plot,year }} \sim \operatorname{Binomial}\left(N 1_{\text {plot,year }}, p_{\text {plot,year }}\right)
$$

and process model,

$$
\begin{aligned}
\operatorname{logit}\left(p_{\text {plot,year }}\right)= & \beta 1+\beta 2 G_{P \text { plot,year }}+\beta 3_{\text {habitat }_{\text {light }}} \text { plot,year } \\
& +\beta 4 \text { soil moisture } \\
& +\beta 5 M_{p-\text { AMFt,year }} \\
& +\beta 6 M_{p-\text { EMFplot,year }} .
\end{aligned}
$$

Given the number of parameters and random effects included, we used a Bayesian approach to estimate them (Clark 2005). Thus parameters were estimated from probability distributions, all with non-informative parameter values, allowing the data to have most of the weight in the estimations (see Appendices C and D for detailed description and model fits). We ran Markov chain Monte Carlo (MCMC) simulations in OpenBuGS (Thomas et al. 2006) to estimate the parameters (50000 iterations). Three chains were monitored for convergence, and after the burn-in period was discarded ( $\sim 2000)$ and chains were thinned to eliminate autocorrelation in the chains (every 100th iteration), we calculated posterior means, standard deviations, and 95\% credible intervals (CI).

Integrated assessment.-We then used these parameter estimates (means, variances, and covariances) to predict survival along gradients of light and soil moisture in both canopy and gap habitats (Eq. 7). Mycorrhizal fungal colonization and growth rate predictions for this simulation were included at the species level using the means and variances of the plotlevel random effects $\left(\omega 1_{\mathrm{m}}, \alpha 1_{\mathrm{m}}, \sigma_{\omega}^{2}\right.$, and $\left.\sigma_{\alpha}^{2}\right)$. Total soil nitrogen content and plant size were set at average plot and species levels. To compare the outcome of our integrated assessment with a null model, we estimated the probability of survival, $p$, as a direct function of resources, $\operatorname{logit}\left(p_{\text {plot,year }}\right)=\beta 1_{\text {null }}+\beta 2_{\text {null }}$ light $_{\text {plot,year }}+$

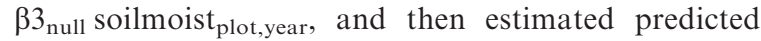
survival along light and soil moisture gradients, maintaining the other variable constant at average level.

\section{RESUlts}

Our experimental design provided a large range of variability in light levels, from $4 \%$ to $93 \%$ of full sunlight and, although gap plots were set up in sites with open 
TABLE 3. Results of parameter estimates, posterior means \pm SD, and $95 \%$ credible intervals (CI) for each of the three sub-models included in the integrated assessment and in the survival null model.

\begin{tabular}{|c|c|c|c|c|c|c|c|c|}
\hline \multirow[b]{2}{*}{ Parameters } & \multicolumn{2}{|c|}{ A. saccharum } & \multicolumn{2}{|c|}{ N. sylvatica } & \multicolumn{2}{|c|}{ C. glabra } & \multicolumn{2}{|c|}{ Q. rubra } \\
\hline & Mean $\pm \mathrm{SD}$ & $95 \% \mathrm{CI}$ & Mean $\pm \mathrm{SD}$ & $95 \% \mathrm{CI}$ & Mean $\pm \mathrm{SD}$ & $95 \% \mathrm{CI}$ & Mean $\pm \mathrm{SD}$ & $95 \% \mathrm{CI}$ \\
\hline \multicolumn{9}{|c|}{ Mycorrhizal colonization, $\varphi$ probability of zero colonization } \\
\hline \multicolumn{9}{|c|}{ Intercept, mean } \\
\hline $\begin{array}{l}\omega 1_{\mathrm{m}} \text { AMF } \\
\omega 1_{\mathrm{m}} \text { EMF }\end{array}$ & $-0.78 \pm 0.53$ & $-1.96,0.14$ & $-2.78 \pm 1.52$ & $-6.7,0.16$ & $\begin{array}{l}-1.83 \pm 0.21 \\
-2.43 \pm 0.68\end{array}$ & $\begin{array}{l}-2.29,-1.46 \\
-3.82,-1.58\end{array}$ & $\begin{array}{l}-42.4 \pm 27 \\
-2.95 \pm 0.75\end{array}$ & $\begin{array}{l}-79.6,-4.4 \\
-4.83,-1.83\end{array}$ \\
\hline \multicolumn{9}{|c|}{ Intercept, variance } \\
\hline $\begin{array}{l}\sigma_{\omega}^{2} \mathrm{AMF} \\
\sigma_{\omega}^{2} \mathrm{EMF}\end{array}$ & $7.64 \pm 4.52$ & $1.73,18.6$ & $6.63 \pm 3.8$ & $1.34,15$ & $\begin{array}{l}0.28 \pm 0.39 \\
2.87 \pm 3.12\end{array}$ & $\begin{array}{r}0.005,1.33 \\
0.08,12.13\end{array}$ & $\begin{array}{l}137 \pm 2113 \\
4.26 \pm 3.6\end{array}$ & $\begin{array}{c}0.1,770 \\
0.98,15.4\end{array}$ \\
\hline \multicolumn{9}{|c|}{ Effect of plant height } \\
\hline $\begin{array}{l}\omega 2 \mathrm{AMF} \\
\omega 2 \mathrm{EMF}\end{array}$ & $0.48 \pm 0.06$ & $0.35,0.58$ & $0.04 \pm 0.16$ & $-0.2,0.4$ & $\begin{array}{l}-0.54 \pm 0.33 \\
-0.53 \pm 0.3\end{array}$ & $\begin{array}{l}-1.04,0.13 \\
-1.09,0.11\end{array}$ & $\begin{array}{l}-0.92 \pm 2.7 \\
-0.56 \pm 0.33\end{array}$ & $\begin{array}{l}-5.7,3.12 \\
-1.3,0.07\end{array}$ \\
\hline \multicolumn{9}{|c|}{ Random effects variance } \\
\hline $\begin{array}{l}\sigma_{\varphi}^{2} \mathrm{AMF} \\
\sigma_{\varphi}^{2} \mathrm{EMF}\end{array}$ & $1.41 \pm 1.3$ & $0.01,4.54$ & $0.06 \pm 0.06$ & $0.004,0.2$ & $\begin{array}{r}0.3 \pm 0.39 \\
0.38 \pm 0.41\end{array}$ & $\begin{array}{l}0.004,1.39 \\
0.007,1.39\end{array}$ & $\begin{array}{l}0.81 \pm 1.53 \\
0.84 \pm 1.47\end{array}$ & $\begin{array}{l}0.007,5.88 \\
0.006,5.05\end{array}$ \\
\hline \multicolumn{9}{|c|}{ Mycorrhizal abundance, $\ln (\lambda)$ percentage of mycorrzhizal colonization } \\
\hline $\begin{array}{l}R_{\mathrm{AMF}}^{2} \\
R_{\mathrm{EMF}}^{2}\end{array}$ & 0.89 & & 0.94 & & $\begin{array}{l}0.98 \\
0.96\end{array}$ & & $\begin{array}{l}0.99 \\
0.97\end{array}$ & \\
\hline \multicolumn{9}{|c|}{ Intercept, mean } \\
\hline $\begin{array}{l}\alpha 1_{\mathrm{m}} \text { AMF } \\
\alpha 1_{\mathrm{m}} \text { EMF }\end{array}$ & $3.82 \pm 0.24$ & $3.33,4.16$ & $2.61 \pm 0.2$ & $2.2,3$ & $\begin{array}{l}4.02 \pm 0.13 \\
3.77 \pm 0.06\end{array}$ & $\begin{array}{l}6.63,4.24 \\
3.64,3.9\end{array}$ & $\begin{array}{r}2.93 \pm 0.27 \\
3.4 \pm 0.11\end{array}$ & $\begin{array}{l}2.46,3.36 \\
3.19,3.64\end{array}$ \\
\hline \multicolumn{9}{|c|}{ Intercept, variance } \\
\hline $\begin{array}{l}\sigma_{\alpha}^{2} \text { AMF } \\
\sigma_{\alpha}^{2} \text { EMF }\end{array}$ & $0.36 \pm 0.29$ & $0.01,1.13$ & $0.22 \pm 0.11$ & $0.07,0.53$ & $\begin{array}{l}0.95 \pm 0.23 \\
0.28 \pm 0.09\end{array}$ & $\begin{array}{l}0.56,1.47 \\
0.13,0.5\end{array}$ & $\begin{array}{l}0.03 \pm 0.06 \\
0.01 \pm 0.02\end{array}$ & $\begin{array}{l}9 \times 10^{-5}, 0.22 \\
1 \times 10^{-4}, 0.07\end{array}$ \\
\hline \multicolumn{9}{|c|}{ Effect of light CANOPY } \\
\hline $\begin{array}{c}\alpha 2_{\text {canopy }} \\
\text { AMF }\end{array}$ & $-0.03 \pm 0.01$ & $-0.06,-0.01$ & $0.001 \pm 0.01$ & $-0.02,0.01$ & $-0.03 \pm 0.005$ & $-0.04,-0.02$ & $-0.002 \pm 0.005$ & $-0.01,0.007$ \\
\hline $\begin{array}{c}\alpha 2_{\text {canopy }} \\
\text { EMF }\end{array}$ & & & & & $-0.0026 \pm 0.007$ & $-0.01,0.006$ & $-0.001 \pm 0.005$ & $-0.01,0.007$ \\
\hline \multicolumn{9}{|c|}{ Effect of light GAP } \\
\hline$\alpha 2_{\text {gap }}$ & $-0.001 \pm 0.005$ & $-0.01,0.008$ & $0.015 \pm 0.005$ & $0.004,0.02$ & $-0.008 \pm 0.003$ & $-0.01,-0.005$ & $0.0007 \pm 0.004$ & $-0.007,0.007$ \\
\hline$\stackrel{\alpha 2_{\text {gap }}}{\mathrm{EMF}}$ & & & & & $-0.0001 \pm 0.001$ & $-0.003,0.003$ & $-0.007 \pm 0.002$ & $-0.01,-0.002$ \\
\hline \multicolumn{9}{|c|}{ Effect of soil moisture } \\
\hline $\begin{array}{l}\alpha 3 \mathrm{AMF} \\
\alpha 3 \mathrm{EMF}\end{array}$ & $-0.15 \pm 0.01$ & $-0.19,-0.13$ & $-0.02 \pm 0.01$ & $-0.05,0.008$ & $\begin{array}{l}-0.01 \pm 0.006 \\
-0.01 \pm \mathbf{0 . 0 0 4}\end{array}$ & $\begin{array}{l}-0.03,-0.004 \\
-0.02,-0.004\end{array}$ & $\begin{array}{c}-\mathbf{0 . 0 4} \pm \mathbf{0 . 0 1} \\
-0.0003 \pm 0.006\end{array}$ & $\begin{array}{l}-\mathbf{0 . 0 7},-\mathbf{0 . 0 2} \\
-0.01,0.01\end{array}$ \\
\hline \multicolumn{9}{|c|}{ Random effects variance } \\
\hline $\begin{array}{l}\sigma_{\lambda}^{2} \mathrm{AMF} \\
\sigma_{\lambda}^{2} \mathrm{EMF}\end{array}$ & $0.89 \pm 0.3$ & $0.45,1.59$ & $0.63 \pm 0.19$ & $0.33,1.09$ & $\begin{array}{l}0.08 \pm 0.02 \\
0.21 \pm 0.05\end{array}$ & $\begin{array}{l}0.04,0.13 \\
0.12,0.33\end{array}$ & $\begin{array}{r}1.06 \pm 0.16 \\
0.4 \pm 0.05\end{array}$ & $\begin{array}{r}0.76,1.41 \\
0.3,0.53\end{array}$ \\
\hline \multicolumn{9}{|l|}{ Growth rate } \\
\hline$R^{2}$ & 0.99 & & 0.98 & & 0.36 & & 0.99 & \\
\hline \multicolumn{9}{|c|}{ Maximum growth rates, gmax } \\
\hline$\gamma 1$ intercept & $4.48 \pm 0.4$ & $3.81,5.03$ & $1.47 \pm 0.12$ & $1.22,1.7$ & $12.5 \pm 0.57$ & $11.2,13.4$ & $9.36 \pm 0.21$ & $9.14,9.86$ \\
\hline $\begin{array}{c}\gamma 2, \text { effect } \\
\text { of plant } \\
\text { height }\end{array}$ & $4.19 \pm 0.46$ & $3.33,5.12$ & $0.94 \pm 0.16$ & $0.64,1.28$ & $4.68 \pm 1.18$ & $2.54,7.25$ & $4.78 \pm 0.77$ & $3.3,6.42$ \\
\hline $\begin{array}{l}\sigma_{\text {gmax }}^{2} \\
\text { random } \\
\text { effects }\end{array}$ & $9.8 \pm 2.97$ & $4.98,16.6$ & $0.22 \pm 0.11$ & $0.07,0.53$ & $4.3 \pm 7.8$ & $0.009,28.6$ & $50.3 \pm 13.7$ & $28.01,79.6$ \\
\hline \multicolumn{9}{|c|}{ Compensation point, lo } \\
\hline$\kappa 1$ intercept & $4.09 \pm 0.32$ & $3.49,4.64$ & $0.02 \pm 0.05$ & $\begin{array}{c}1 \times 10^{-6} \\
0.22\end{array}$ & $0.22 \pm 0.34$ & $\begin{array}{c}1.1 \times 10^{-5} \\
0.98\end{array}$ & $0.03 \pm 0.08$ & $\begin{array}{c}9 \times 10^{-6} \\
0.39\end{array}$ \\
\hline $\begin{array}{c}\kappa 2 \text {, effect } \\
\text { of soil } \\
\text { nitrogen }\end{array}$ & $-0.32 \pm 0.39$ & $-1.3,0.39$ & $1.24 \pm 1.6$ & $-0.92,4.76$ & $-3.24 \pm 4.71$ & $-11.6,5.7$ & $-6.17 \pm 4.64$ & $-11.1,4.9$ \\
\hline $\begin{array}{l}\sigma_{\text {lo }}^{2}, \\
\quad \text { random } \\
\quad \text { effects }\end{array}$ & $27.5 \pm 15$ & $0.83,64$ & $1.43 \pm 2.85$ & $0.007,9.63$ & $22.06 \pm 47.3$ & $0.01,157$ & $57.8 \pm 20.6$ & $25.5,104$ \\
\hline \multicolumn{9}{|c|}{ Half saturation point, $\theta$} \\
\hline$\stackrel{\mu 1}{\text { intercept }}$ & $4.59 \pm 0.41$ & $3.89,5.15$ & $0.92 \pm 0.1$ & $0.75,1.15$ & $0.007 \pm 0.008$ & $\begin{array}{c}7 \times 10^{-6} \\
0.02\end{array}$ & $0.7 \pm 0.08$ & $0.52,0.86$ \\
\hline $\begin{array}{l}\mu 2 \text {, effect } \\
\text { of AMF } \\
\text { fungi }\end{array}$ & $0.53 \pm 0.12$ & $0.33,0.67$ & $0.78 \pm 0.3$ & $0.37,1.56$ & $-0.77 \pm 0.07$ & $-0.95,-0.68$ & $-0.03 \pm 0.03$ & $-0.09,0.03$ \\
\hline
\end{tabular}


TABle 3. Continued.

\begin{tabular}{|c|c|c|c|c|c|c|c|c|}
\hline \multirow[b]{2}{*}{ Parameters } & \multicolumn{2}{|c|}{ A. saccharum } & \multicolumn{2}{|c|}{ N. sylvatica } & \multicolumn{2}{|c|}{ C. glabra } & \multicolumn{2}{|c|}{ Q. rubra } \\
\hline & Mean \pm SD & $95 \% \mathrm{CI}$ & Mean \pm SD & $95 \% \mathrm{CI}$ & Mean \pm SD & $95 \% \mathrm{CI}$ & Mean \pm SD & $95 \% \mathrm{CI}$ \\
\hline $\begin{array}{l}\mu 3 \text {, effect } \\
\text { of EMF } \\
\text { fungi }\end{array}$ & & & & & $2.12 \pm 0.21$ & $1.81,2.69$ & $0.32 \pm 0.05$ & $0.26,0.42$ \\
\hline $\begin{array}{l}\sigma_{\theta}^{2}, \\
\quad \text { random } \\
\text { effects }\end{array}$ & $8.3 \pm 17$ & $0.004,63$ & $2.3 \pm 3.16$ & $0.09,11.8$ & $221 \pm 309$ & $0.21,1048$ & $1.11 \pm 4.22$ & $0.006,8.7$ \\
\hline \multicolumn{9}{|c|}{ Effect of soil moisture } \\
\hline$\delta$ & $0.23 \pm 0.007$ & $0.22,0.24$ & $0.08 \pm 0.004$ & $0.07,0.088$ & $0.59 \pm 0.01$ & $0.56,0.62$ & $0.49 \pm 0.01$ & $0.47,0.51$ \\
\hline Random effect & & & & & & & & \\
\hline$\sigma_{\mathrm{G}}^{2}$ & $0.52 \pm 0.49$ & $0.009,1.7$ & $0.08 \pm 0.05$ & $0.008,0.2$ & $16.7 \pm 3.3$ & $10.9,24$ & $4.05 \pm 3.42$ & $0.05,12.3$ \\
\hline \multicolumn{9}{|c|}{ Survival integrated model } \\
\hline$R^{2}$ & 0.80 & & 0.95 & & 0.85 & & 0.86 & \\
\hline$\beta 1$, intercept & $1.07 \pm 0.6$ & $-0.26,2.42$ & $1.62 \pm 2.29$ & $-2.49,6.51$ & $-3.65 \pm 1.6$ & $-6.95,-0.75$ & $3.07 \pm 3.41$ & $-4.34,8.73$ \\
\hline $\begin{array}{l}\beta 2 \text {, effect of } \\
\text { growth } \\
\text { rate }\end{array}$ & $0.57 \pm 0.21$ & $0.08,0.96$ & $0.8 \pm 2.4$ & $-4.4,5.1$ & $0.97 \pm 0.2$ & $0.66,1.46$ & $0.13 \pm 0.21$ & $-0.43,0.45$ \\
\hline $\begin{array}{l}\beta 33_{\text {canopy, }} \\
\text { effect of } \\
\text { light }_{\text {CANOPY }}\end{array}$ & $-0.04 \pm 0.02$ & $-0.08,-0.0004$ & $-0.06 \pm 0.06$ & $-0.2,0.06$ & $-0.1 \pm 0.04$ & $-0.19,-0.02$ & $-0.003 \pm 0.03$ & $-0.07,0.06$ \\
\hline $\begin{array}{l}\beta 3_{\text {gap }} \text {, effect } \\
\text { of light } \\
\text { GAP }\end{array}$ & $-0.04 \pm 0.01$ & $-0.06,-0.02$ & $0.01 \pm 0.04$ & $-0.05,0.13$ & $-0.09 \pm 0.01$ & $-0.13,-0.05$ & $-0.017 \pm 0.017$ & $-0.05,0.018$ \\
\hline $\begin{array}{l}\beta 4, \text { effect of } \\
\text { soil } \\
\text { moisture }\end{array}$ & $-0.13 \pm 0.07$ & $-0.26,0.005$ & $-0.06 \pm 0.24$ & $-0.54,0.38$ & $-0.68 \pm 0.11$ & $-0.94,-0.5$ & $-0.15 \pm 0.12$ & $-0.36,0.1$ \\
\hline $\begin{array}{l}\beta 5 \text {, effect of } \\
\text { AMF } \\
\text { fungi }\end{array}$ & $-0.08 \pm 0.05$ & $-0.19,0.007$ & $-0.19 \pm 0.09$ & $-0.39,-0.06$ & $-0.05 \pm 0.01$ & $-0.08,-0.03$ & $-0.06 \pm 0.15$ & $-0.36,0.1$ \\
\hline $\begin{array}{l}\beta 6 \text {, effect of } \\
\text { EMF } \\
\text { fungi }\end{array}$ & & & & & $0.15 \pm 0.03$ & $0.09,0.22$ & $-0.08 \pm 0.03$ & $-0.16,-0.008$ \\
\hline \multicolumn{9}{|c|}{ Survival null model } \\
\hline$R^{2}$ & 0.53 & & 0.9 & & 0.87 & & 0.70 & \\
\hline $\begin{array}{l}\beta 1_{\text {null }}, \\
\text { intercept }\end{array}$ & $0.95 \pm 0.47$ & $0.07,2$ & $5.31 \pm 1$ & $3.4,7.7$ & $4.37 \pm 0.65$ & $3.18,5.75$ & $-2.57 \pm 0.39$ & $-3.46,-1.88$ \\
\hline $\begin{array}{c}\beta 2_{\text {null }} \text {, effect } \\
\text { of light }\end{array}$ & $-0.02 \pm 0.004$ & $-0.028,-0.012$ & $-0.05 \pm 0.01$ & $-0.08,-0.03$ & $-0.02 \pm 0.009$ & $-0.04,-0.003$ & $0.02 \pm 0.004$ & $0.01,0.03$ \\
\hline $\begin{array}{l}\beta 3_{\text {null, }} \text { effect } \\
\quad \text { of soil } \\
\text { moisture }\end{array}$ & $0.02 \pm 0.004$ & $-0.06,0.1$ & $-0.46 \pm 0.09$ & $-0.68,-0.29$ & $-0.41 \pm 0.06$ & $-0.54,-0.3$ & $0.19 \pm 0.03$ & $0.13,0.26$ \\
\hline
\end{tabular}

Notes: Subscript $\varphi$ is the probability of not detecting colonization. Fixed effects with statistically significant estimates $(95 \%$ CI does not include zero) are shown in boldface type. $R^{2}$ is the fit of predicted vs. observed. Empty cells indicate that that particular species is not colonized by that particular group of mycorrhizal fungi.

canopies, many had light levels as low as those of the canopy plots due to low vegetation shading the ground (i.e., tree saplings). Volumetric soil moisture varied from $1 \%$ to $19.5 \%$, and total soil nitrogen ranged from 24.9 to $311 \mathrm{mg} / \mathrm{kg}$. There was a substantial overlap between the two regions in light, soil moisture, and nitrogen levels (see Appendix A).

In general, all model fits, predicted vs. observed, were high $\left(R^{2}>0.8\right)$, except for the Carya glabra growth submodel $\left(R^{2}=0.36\right.$; Table 3, Appendix D).

Mycorrhizal fungal colonization ( $\varphi$; Table 3; Fig. 1).- There were no significant differences among plots or years, this included comparisons between the northern and southern regions, among the six forest types and the two habitats ( $\omega 1_{\text {plot,year; }}$ see Appendix D). Analysis of the residuals did not show any differences between seed sources (predicted minus observed, not shown). The overall probabilities of detecting colonization by mycorrhizal fungi $\left(1-\left[e^{\omega 1 \mathrm{~m}} /\left(1+e^{\omega 1 \mathrm{~m}}\right)\right]\right)$ varied among species. All four species were colonized by AMF, with the following probabilities: Acer saccharum 0.68
(95\% CI 0.47-0.87), Nyssa sylvatica 0.94 (95\% CI $0.6-$ $0.99 \%$ ), Carya glabra 0.86 (95\% CI 0.81-0.9), and Quercus rubra 0.99 (95\% CI 0.98-1). Probability of being colonized by EMF was $0.91(0.82-0.97)$ for $C$. glabra and $0.95(0.86-0.99)$ for $Q$. rubra. Initial plant height influenced the probability of mycorrhizal fungal colonization in $A$. saccharum; taller seedlings had a lower probability (positive parameters) of being colonized by AMF $(\omega 2)$.

Mycorrhizal fungal abundance ( $\lambda$; Table 3 ; Fig. 1).We did not find any differences among plots, years, forest types, and habitats on percent mycorrhizal colonization ( $\alpha 1_{\text {plot,year }}$; Appendix D), nor between seed sources (predicted minus observed, analysis of the residuals not shown). Percent AMF colonization declined with increasing light availability for $A$. saccharum seedlings in the canopy (negative parameter $\alpha 2_{\text {canopy }}$ ) and $C$. glabra, in both canopy and gap plots (negative parameters $\alpha 2_{\text {canopy }}$ and $\alpha 2_{\text {gap }}$ ), whereas the opposite pattern occurred for $N$. sylvatica seedlings in the gap plots. EMF abundance decreased with increased light in 

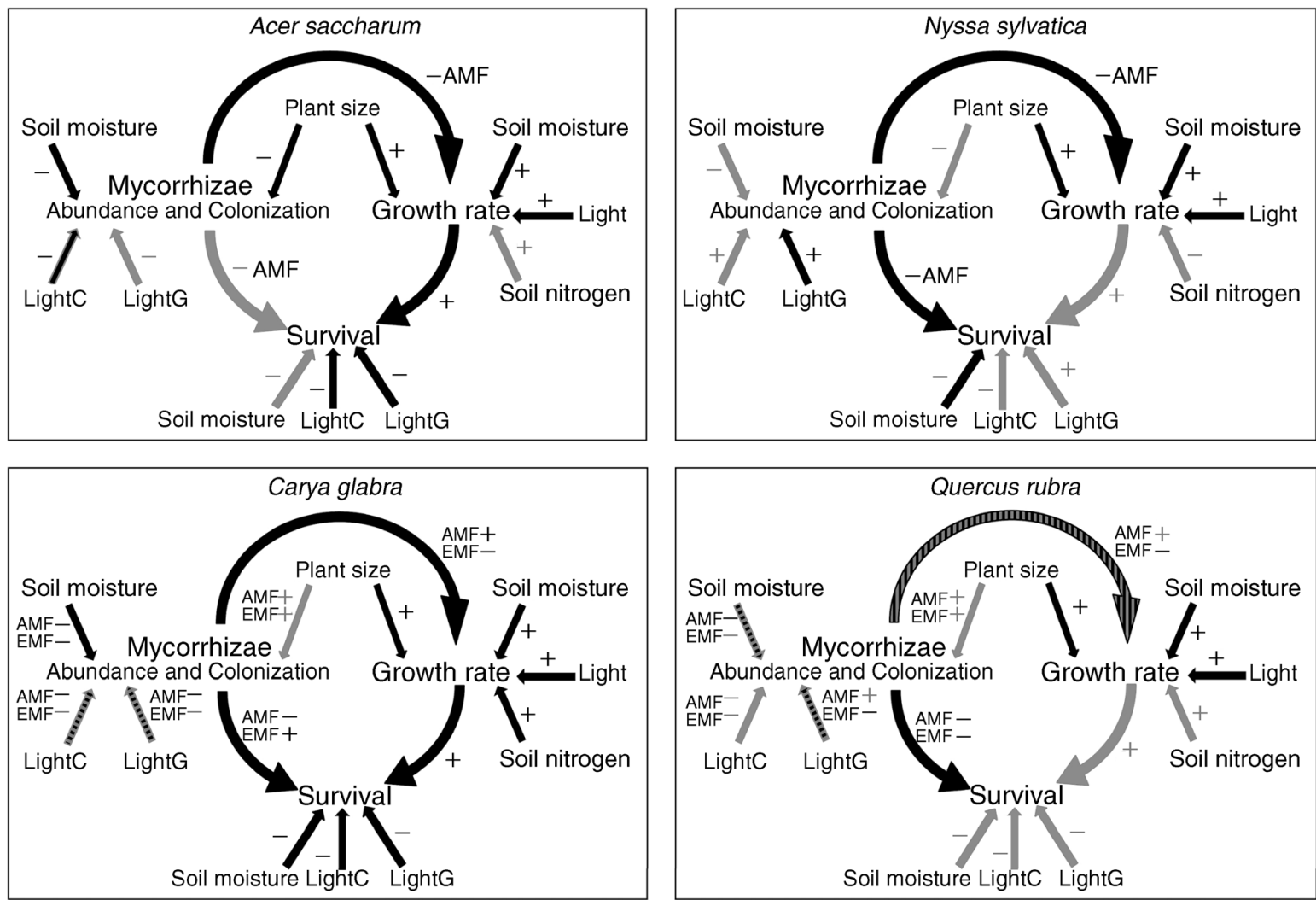

FIG. 1. Graphical representation of the integrated assessment and summary of the results from each of the sub-models: mycorrhizal fungal colonization, seedling growth, and survival during the first growing season. Plus signs $(+)$ indicate increase in mycorrhizal colonization, growth rate, and survival; minus signs (-) reflect the opposite pattern. Abbreviations are: LightC, light in the canopy; LightG, light in the gap. Black arrows indicate statistically significant results $(95 \%$ CI of fixed effects coefficients did not include zero), gray arrows reflect nonsignificant results, gray and black striped arrows indicate that both statistically significant and nonsignificant responses are reported. The effects of light on growth were positive as part of the model configuration.

Q. rubra seedlings when growing in the gap habitat. Soil moisture content, (effect described by parameter $\alpha 3$ ), had a negative effect on AMF abundance in $A$. saccharum, C. glabra, and Q. rubra, and for EMF in C. glabra.

Growth rates (Table 3; Fig. 1).--Maximum growth rates differed greatly among species mainly reflecting the differences in seed size $(\gamma 1)$, and ranged from 4.48 and $1.47 \mathrm{mg} / \mathrm{d}$ for $A$. saccharum and $N$. sylvatica respectively, to 12.5 and $9.36 \mathrm{mg} / \mathrm{d}$ for C. glabra and Q. rubra. Seedlings of all species that were initially tall grew significantly faster (positive values of parameter $\gamma 2$ ). Total soil nitrogen reduced (a positive effect) the compensation point for of C. glabra (negative value of $\kappa 2)$. The abundance of AMF mycorrhizal fungi $(\mu 2)$ increased the half-saturation point (negative effect on growth) in A. saccharum and N. sylvatica and reduced it (positive effect on growth) for C. glabra. Higher EMF abundance contributed to a higher half saturation point for both C. glabra and Q. rubra (negative effect, positive value of parameter $\mu 3$ ). In addition, seedlings of all species grew significantly faster in wetter soils (parameter $\delta$ ).
Survival (Table 3; Fig. 1).-A. saccharum and $C$. glabra seedlings had higher survival in plots with high growth rates (positive parameter $\beta 2$ ). These species, however, had decreased survival as light levels increased in both canopy and gap plots (negative parameters $\beta 3_{\text {canopy }}$ or $\beta 3_{\text {gap }}$ ). Wetter soils were associated with reduced survival of $N$. sylvatica and C. glabra seedlings (negative parameter $\beta 4$ ). The association of mycorrhizal fungi with plot survival varied species to species (as reflected by the sign of parameters $\beta 5$ and $\beta 6$ ). Increasing AMF colonization was related to decreased survival of N. sylvatica, C. glabra, and Q. rubra. Q. rubra had a similar pattern with respect to EMF colonization, while higher survival of C. glabra was associated with higher EMF colonization.

Integrated assessment and the null model.-The simulations integrated all the effects reported here (Fig. 1) into a survival curve for each of the two habitats considered, canopy and gap. Results varied among species illustrating the pattern of the response to increasing light and soil moisture levels and covering most of the variability observed in the data, which the null model did not (Figs. 2 and 3). The light results also 

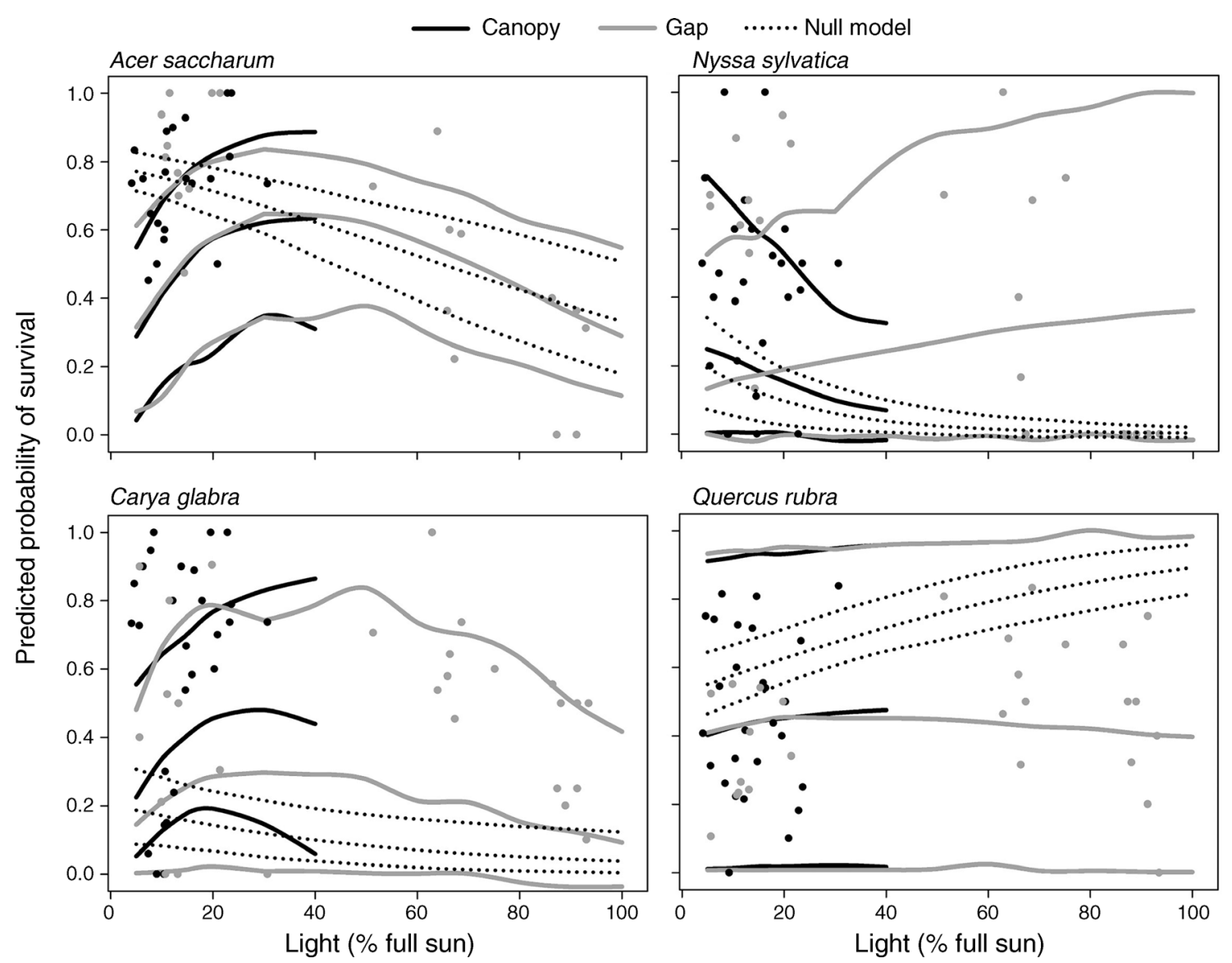

FIG. 2. Predicted survival of tree species recruitment along a light gradient (at average levels of soil moisture and soil nitrogen and average seedling height). Circles represent the data: plot-level survival under the canopy (black dots) and in the gaps (gray dots). Predicted survival curves (means [middle lines] and 95\% predicted intervals [upper and lower lines]) in canopy habitat (black lines, for the range of light values with available data) and gap habitat (gray lines). Dotted lines represent predicted survival from the null model (at average soil moisture).

point at differential signs and thresholds in the response, A. saccharum and C. glabra showed a threshold in their responses to increasing light, with increasing survival as irradiance increased until reaching a threshold around $\sim 20-30 \%$ full sun, after which survival decreases with increasing light levels (Fig. 2). The overall, direct (as part of the survival model) and indirect (through the growth and mychorrizal fungi models and their effects on survival), effect of increasing light level on $N$. sylvatica survival was negative in the canopy and positive in the gap (Fig. 2). Q. rubra showed an almost flat response to light (Fig. 2). The outcome from the null model indicates a negative effect of light on three species and a positive effect on one, Q. rubra (Table 3; Fig. 2). Trends in predicted survival along the soil moisture gradient (Fig. 3) were almost flat for three species, only C. glabra showed a clear decline in survival with increasing soil moisture levels. The null model showed clear negative effects for all species except for $A$. saccharum (Fig. 3).

\section{Discussion}

We used first-year demographic data together with an integrated analytical framework to assess recruitment dynamics of four tree species. Although we did not investigate the specific physiological pathways underlying particular relationships, we did quantify the extent of those relationships, and integrate them into a comprehensive assessment of tree recruitment (Fig. 1). We observed a complex set of responses to resources that cannot be explained by simple resource availability mechanisms. This integrated analysis led us to account for most of the variability observed in the data in addition to letting us identify the patterns and thresholds in the species' recruitment dynamics along resource gradients (Figs. 2 and 3). Also, by including individual variability, i.e., random effects, at different steps of the process, we not only quantified the species response but also the observed individuals' variability around those responses.

Light is a major driver of tree species recruitment in temperate forests (Pacala et al. 1996). With respect to 


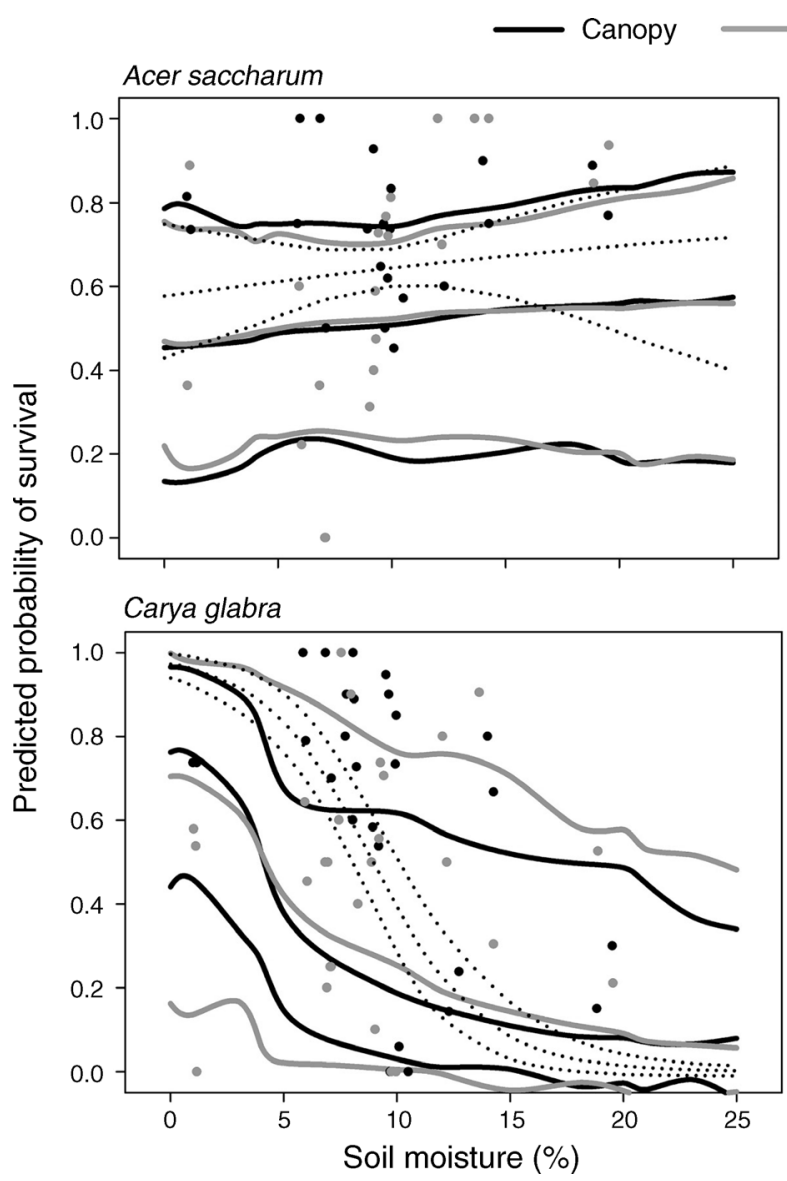

Gap $\quad \cdots . .$. Null model

Nyssa sylvatica

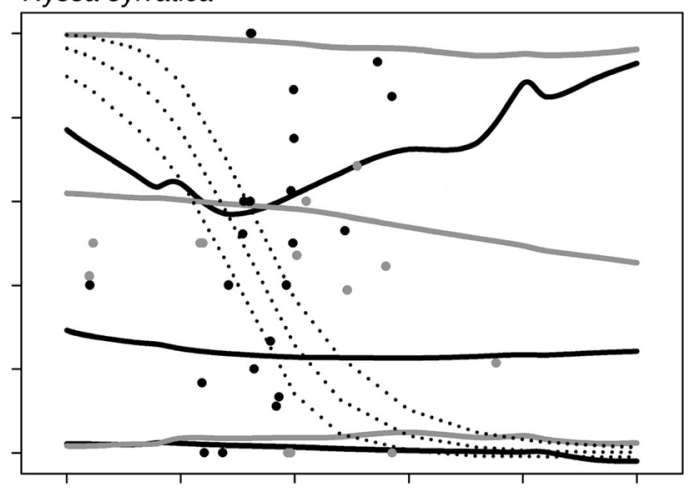

Quercus rubra

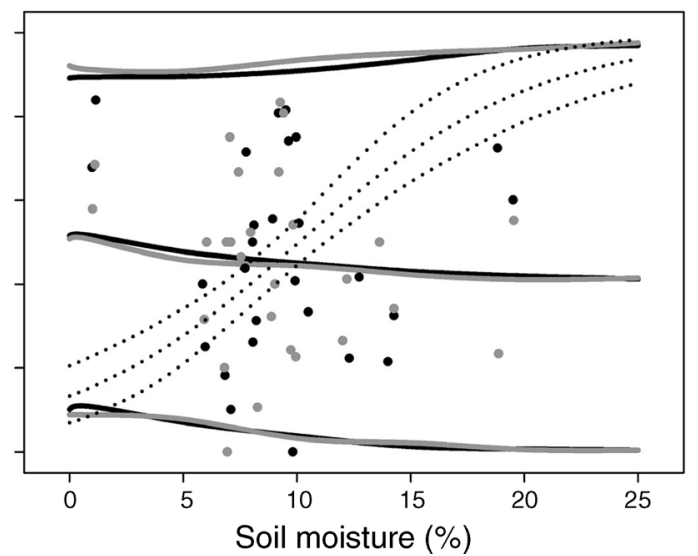

FIG. 3. Predicted survival of tree species recruitment along a soil moisture gradient (at average light levels at each habitat, average soil nitrogen, and average seedling height). Circles represent the data: plot-level survival under the canopy (black dots) and in the gaps (gray dots). Predicted survival curves (means [middle lines] and 95\% predicted intervals [upper and lower lines]) in canopy habitat (black lines) and gap habitat (gray lines). Dotted lines represent predicted survival from the null model (at average light at each habitat).

survival and growth, we often assume that increasing light will benefit seedlings (e.g., Canham et al. 1999, Dalling et al. 2004), but this may occur only if we consider the direct impact of light on individuals (i.e., as a resource necessary to fix carbon). However, the conditions created by a particular light environment may drive recruitment patterns both directly, e.g., determining carbon fixation rates, and indirectly, e.g., affecting water availability or competitive pressure. Our results illustrate how increasing light levels may have contrasting results among species, and within a species between habitats. For A. saccharum and C. glabra, increasing light had a positive effect at low levels, but both species reached a point where the combined direct and indirect effects of light became detrimental for survival (Fig. 2). Such a pattern is likely the result of competitive pressure from the ground layer, forbs and grasses that thrive at high light and may outcompete tree seedlings for other resources (e.g., Fahey and Puettmann 2008, Parker et al. 2009), making high-light environ- ments less suitable for seedling survival even if higher light levels per se would benefit individual performance.

Other factors may also indirectly shape the effects of a particular light environment on seedling performance. For example, the availability of soil nutrients and water can increase seedling survival at low light levels (Walters and Reich 1997). In our analysis, we accounted for differences in soil total nitrogen when estimating the compensation point, the light level at which seedlings start to grow, but only one species, C. glabra, benefited from higher nitrogen in the soil. The observed effects of soil moisture on mycorrhizal fungal colonization and seedling survival resulted in unexpected outcomes that might be explained by the interaction between water and light (Fig. 1). When considered in conjunction with other intervening factors, the effects of soil moisture were mainly negative. We speculate this pattern is due to interactions with soil pathogens, more prevalent in high moisture sites, and low carbon fixation rates, at more humid low light environments (Augspurger 1990, Reinhart et al. 2010). 


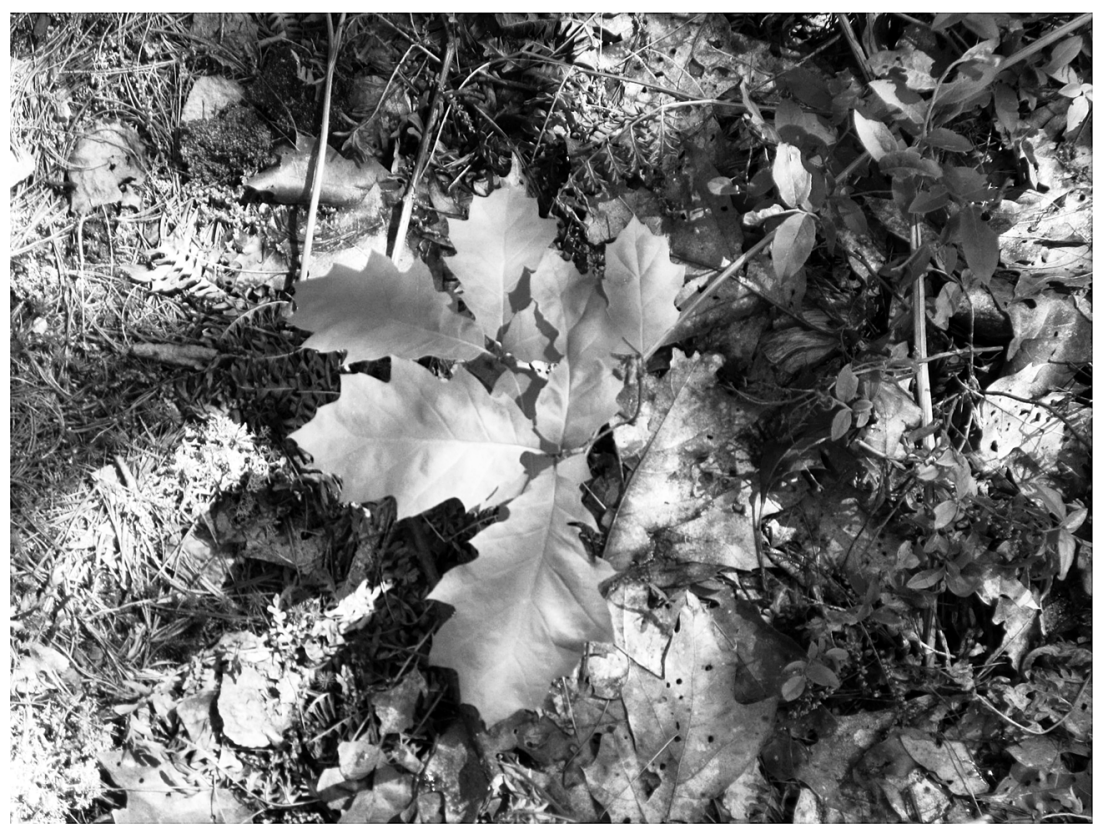

Plate 1. Seedling of Quercus rubra growing in one of the experimental plots. Photo credit: I. Ibáñez.

Light also drives the symbiosis between seedlings and mycorrhizal fungi, as the effect of the symbiosis (positive or negative) will depend on excess carbon from the plant (Gehring and Connell 2006, Grman 2012). The patterns of mychorrizal fungal colonization we observed among seedlings seem to indicate that certain thresholds of seed reserves or carbon fixation are needed for the symbiosis to take place. Mycorrhizal fungi also played a role in seedling's growth response to increasing light levels, varying from negative to positive (Table 3; Fig. 1). These results again point at the combined effects of interacting variables, light and mycorrhizal fungal colonization, and specifically illustrate the potential changing role of mycorrhizal symbiosis on growth, from beneficial to detrimental, depending on the light environment (Moora and Zobel 1998, van der Heijden and Horton 2009).

Although lack of mycorrhizal symbiosis and consequent failure to establish has been reported before for tree species (e.g., Thiet and Boerner 2007, Nuñez et al. 2009) we observed many healthy seedlings without obvious mycorrhizal fungal colonization (up to $49 \%$ for A. saccharum). In our study, once resource levels were accounted for, we did not find regional, stand, canopy, or year differences affecting the probability of mycorrhizal fungal colonization of the roots, nor the abundance of mycorrhizae on those seedlings that had established the symbiosis (see Appendix D). These results seem to point to a diverse mycorrhizal fungal community in forested stands that would interact with tree seedlings regardless of conspecific adult trees being present or not, e.g., south species planted in the northern plots or species planted in different forest types. Among those seedlings with mycorrhizae, AMF colonization was always associated with reduced survival, while the association with EMF colonization varied. Also, larger seedlings of $A$. saccharum, a small-seeded species, had lower probabilities of being colonized by AMF, a pattern that may be reflecting, to some extent, the first year seedlings' capacity to prevent colonization if detrimental (e.g., Wallenda and Kottke 1998, Lilleskov et al. 2002).

Conclusions. - Even though we only accounted for a small fraction of the variables affecting recruitment and applied very simple, mostly linear, relationships, our integrated assessment (Fig. 1) identified much more complex recruitment dynamics than what would have been predicted from direct mechanisms (the null model). Integrating all the information available on seedling recruitment into a comprehensive analysis of seedling survival allowed us to discern realistic responses to resource levels, to identify threshold along those responses and to account for most of the variability in survival observed in the data. From the physiological perspective of an individual seedling, an increase in resource level should always have a positive or saturating effect on individual performance (within its physiological tolerance range). However an increase in resources, in this case light and soil moisture, does not take place in isolation, but is part of a larger context where the number of driving factors, their interactions and combined effects are all affecting that individual. Our integrated assessment, although still relatively simple, represented that larger context. Species' response to increasing light was not always positive and went through thresholds that switched the nature of the 
relationship from positive to negative, and the overall response to increasing soil moisture was mostly negative. It is unlikely that these patterns reflect a direct negative effect of light or soil moisture, but instead a combined effect that accounts for other indirect pathways, e.g., increase in competition from other species, incidence of pathogens or detrimental effects of mycorrhizal fungi (e.g., AMF). In summary, a holistic approach to tree species recruitment dynamics allowed us to account for the wide range of responses reflected in our data and brought a better understanding of forest regeneration than just the sum of the parts, as interactions and feedbacks were indirectly accounted for and integrated into our estimates.

\section{ACKNOWLEDGMENTS}

We are grateful to the GCEL, PEDG, and Kobe lab groups at the University of Michigan and Michigan State University, and to B. Ibáñez and E. Holste for helpful comments on the manuscript. We thank the numerous assistants who helped throughout the experiment. This research was funded by the National Science Foundation (DEB-EAGER 0947783) and the USDA McIntire-Stennis Program (USDA MICY000706).

\section{Literature Cited}

Ackerly, D. D., and F. A. Bazzaz. 1995. Plant growth and reproduction along $\mathrm{CO} 2$ gradients: non-linear responses and implications for community change. Global Change Biology 1:199-207.

Augspurger, C. K. 1990. Spatial patterns of damping-off diseases during seedling recruitment in tropical forests. Pages 131-144 in J. J. Burdon and S. R. Leather, editors. Pests, pathogens and plant communities. Blackwell Scientific Publications, Oxford, UK.

Barnes, B. V., and W. H. Wagner. 2007. Michigan trees. University of Michigan Press, Ann Arbor, Michigan, USA.

Beckage, B., J. S. Clark, B. D. Clinton, and B. L. Haines. 2000. A long-term study of tree seedling recruitment in southern Appalachian forests: the effects of canopy gaps and shrub understories. Canadian Journal of Forest Research 30:16171631.

Bereau, M., D. Bonal, E. Louisanna, and J. Garbaye. 2005. Do mycorrhizas improve tropical tree seedling performance under water stress and low light conditions? A case study with Dicorynia guianensis (Caesalpiniaceae). Journal of Tropical Ecology 21:375-381.

Caldwell, J. M., E. I. Sucoff, and R. K. Dixon. 1995. Grass interference limits resource availability and reduces growth of juvenile red pine in the field. New Forests 10:1-15.

Canham, C. D., R. K. Kobe, E. F. Latty, and R. L. Chazdon. 1999. Interspecific and intraspecific variation in tree seedlings survival: effects of allocation to roots versus carbohydrates reserves. Oecologia 121:1-11.

Clark, J. S. 2005. Why environmental scientists are becoming Bayesians. Ecology Letters 8:2-14.

Clark, J. S., J. Mohan, M. Dietze, and I. Ibáñez. 2003. Coexistence: how to identify trophic trade-offs. Ecology 84:17-31.

Comita, L. S., M. Uriarte, J. Thompson, I. Jonckheere, C. D. Canham, and J. K. Zimmerman. 2009. Abiotic and biotic drivers of seedling survival in a hurricane-impacted tropical forest. Journal of Ecology 97:1346-1359.

Dalling, J. W., K. Winter, and S. P. Hubbell. 2004. Variation in growth responses of neotropical pioneers to simulated forest gaps. Functional Ecology 18:725-736.

Fahey, R. T., and K. J. Puettmann. 2008. Patterns in spatial extent of gap influence on understory plant communities. Forest Ecology and Management 255:2801-2810.
Gehring, C. A., and J. H. Connell. 2006. Arbuscular mycorrhizal fungi in the tree seedlings of two Australian rain forests: occurrence, colonization, and relationships with plant performance. Mycorrhiza 16:89-98.

Gelfand, A. E., and S. K. Ghosh. 1998. Model choice: a minimum posterior predictive loss approach. Biometrika 85: $1-11$.

Grman, E. 2012. Plant species differ in their ability to reduce allocation to non-beneficial arbuscular mycorrhizal fungi. Ecology 93:711-718.

Grubb, P. J. 1977. The maintenance of species-richness in plant communities: the importance of the regeneration niche. Biological Review, Cambridge Philosophycal Society 52: 102-145.

Gurevitch, J. S., M. Scheiner, and G. A. Fox. 2006. The ecology of plants. Second edition. Sinauer Associates, Sunderland, Massachusetts, USA.

Ibáñez, I., J. S. Clark, and M. C. Dietze. 2008. Evaluating the sources of potential migrant species. Implications under climate change. Ecological Applications 18:1664-1678.

Ibáñez, I., J. S. Clark, and M. C. Dietze. 2009. Estimating colonization potential of migrant tree species. Global Change Biology 5:1173-1188.

Ibáñez, I., J. S. Clark, S. L. LaDeau, and J. Hille Ris Lambers. 2007. Exploiting temporal variability to understand tree recruitment response to climate change. Ecological Monographs 77:163-177.

Jin, L., S. H. Wang, X. J. Wang, and Y. Y. Shen. 2009. Seed size influences arbuscular mycorrhizal symbiosis across leguminous host-plant species at the seedling stage. Symbiosis 49:111-116.

Johnson, N. C., J. H. Graham, and F. A. Smith. 1997. Functioning of mycorrhizal associations along the mutualism-parasitism continuum. New Phytologist 135:575-586.

Kaelke, C. M., E. L. Kruger, and P. B. Reich. 2001. Trade-offs in seedling survival, growth, and physiology among hardwood species of contrasting successional status along a lightavailability gradient. Canadian Journal of Forest Research 31:1602-1616.

Lilleskov, E. A., T. J. Fahey, T. R. Horton, and G. M. Lovett. 2002. Belowground ectomycorrhizal fungal community change over a nitrogen deposition gradient in Alaska. Ecology 83:104-115.

Löf, M. 2000. Establishment and growth in seedlings of Fagus sylvatica and Quercus robur: influence of interference from herbaceous vegetation. Canadian Journal of Forest Research 30:855-864.

McCarthy-Neumann, S., and I. Ibáñez. 2012. Tree range expansion may be enhanced by escape from negative plantsoil feedbacks. Ecology 93:2637-2649.

McGonigle, T. P., and A. H. Fitter. 1990. Ecological specificity of vesicular-arbuscular mycorrhizal associations. Mycological Research 94:120-122.

Moora, M., and M. Zobel. 1998. Arbuscularmycorrhiza and plant-plant interactions: impact of invisible world on visible patterns. Pages 79-98 in F. I. Pugnaire, editor. Positive interactions and plant community dynamics. CRC Press, Boca Ration, Florida, USA.

Myers, J. A., and K. Kitajima. 2007. Carbohydrate storage enhances seedling shade and stress tolerance in a neotropical forest. Journal of Ecology 95:383-395.

Nuñez, M. A., T. R. Horton, and D. Simberloff. 2009. Lack of belowground mutualisms hinders Pinaceae invasions. Ecology 90:2352-2359.

O'Connor, M. I., E. R. Selig, M. L. Pinsky, and F. Altermatt. 2012. Toward a conceptual synthesis for climate change responses. Global Ecology and Biogeography 21:693-703.

Pacala, S. W., C. D. Canham, J. Saponara, J. A. Silander, R. K. Kobe, and E. Ribbens. 1996. Forest models defined by field measurements: estimation, error analysis and dynamics. Ecological Monographs 66:1-43. 
Parker, W. C., D. G. Pitt, and A. E. Morneault. 2009. Influence of woody and herbaceous competition on microclimate and growth of eastern white pine (Pinus strobus L.) seedlings planted in a central Ontario clearcut. Forest Ecology and Management 258:2013-2025.

Piper, F. I., M. Reyes-Diaz, L. J. Corcuera, and C. H. Lusk. 2009. Carbohydrate storage, survival, and growth of two evergreen Nothofagus species in two contrasting light environments. Ecological Research 24:1233-1241.

Reinhart, K. O., A. A. Royo, S. A. Kageyama, and K. Clay. 2010. Canopy gaps decrease microbial densities and disease risk for a shade-intolerant tree species. Acta Oecologica 36: 530-536.

Rich, P. M., D. B. Clark, D. A. Clark, and S. F. Oberbauer. 1993. Long-term study of solar radiation regimes in a tropical wet forest using quantum sensors and hemispherical photography. Agricultural and Forest Meteorology 65:107-127.

Suarez, M. L., and T. Kitzberger. 2008. Recruitment patterns following a severe drought: long-term compositional shifts in Patagonian forests. Canadian Journal of Forest Research 38: 3002-3010.

Teste, F. P., S. W. Simard, D. M. Durall, R. D. Guy, M. D. Jones, and A. L. Schoonmaker. 2009. Access to mycorrhizal networks and roots of trees: importance for seedling survival and resource transfer. Ecology 90:2808-2822.

Thiet, R. K., and R. E. J. Boerner. 2007. Spatial patterns of ectomycorrhizal fungal inoculum in arbuscular mycorrhizal barrens communities: implications for controlling invasion by Pinus virginiana. Mycorrhiza 17:507-517.
Thomas, A., R. O'Hara, U. Ligges, and S. Sturts. 2006. Making BUGS open. R News 6:12-17.

Uriarte, M., J. S. Clark, J. K. Zimmerman, L. S. Comita, J. Forero-Montana, and J. Thompson. 2012. Multidimensional trade-offs in species responses to disturbance: implications for diversity in a subtropical forest. Ecology 93:191205.

van der Heijden, M. 1. G. A., and T. R. Horton. 2009. Socialism in soil? The importance of mycorrhizal fungal networks for facilitation in natural ecosystems. Journal of Ecology 97: $1139-1150$.

Vierheilig, H., A. P. Coughlan, U. Wyss, and Y. Piche. 1998. Ink and vinegar, a simple staining technique for arbuscularmycorrhizal fungi. Applied and Environmental Microbiology 64:5004-5007.

Wallenda, T., and I. Kottke. 1998. Nitrogen deposition and ectomycorrhizas. New Phytologist 139:169-187.

Walters, M. B., and P. B. Reich. 1997. Growth of Acer saccharum seedlings in deeply shaded understories of northern Wisconsin: effects of nitrogen and water availability. Canadian Journal of Forest Research 27:237-247.

Zangaro, W., F. R. Nishidate, F. R. S. Camargo, G. G. Romagnoli, and J. Vandressen. 2005. Relationships among arbuscular mycorrhizas, root morphology and seedling growth of tropical native woody species in southern Brazil. Journal of Tropical Ecology 21:529-540.

Zuur, A. F., E. N. Ieno, N. J. Walker, A. A. Saveliev, and G. M. Smith. 2009. Mixed effects models and extensions in ecology with R. Springer Science+Business Media, New York, New York, USA.

\section{Supplemental Material}

\section{Appendix A}

Summary of environmental data: light, soil moisture, and soil nitrogen (Ecological Applications E095-032-A1).

\section{Appendix B}

Exploratory data analysis (correlations) used for model configuration (Ecological Applications E095-032-A2).

\section{Appendix C}

Analytical methods: detailed description of the model and parameter estimation (Ecological Applications E095-032-A3).

Appendix D

Additional results: models' fits and plot and year random effects (Ecological Applications E095-032-A4).

\section{Supplement}

OpenBUGS code for the mychorrhizal fungi colonization, growth, and survival models (Ecological Applications E095-032-S1). 